\title{
VELOCITY GRADIENTS AND MICROTURBULENCE IN CEPHEIDS
}

\section{ALAN H. KARP}

(NASA-TH $-\mathrm{X}-66081)$ MICROTURBULENCE IN CEPHEIDS (NASA)

\section{OCTOBER 1972}

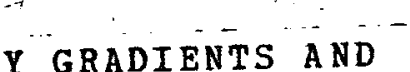

A. H. Karp

$$
\text { CSCL O3A }
$$


Velocity Gradients and Microturbulence in Cepheids

\author{
Alan H. Karp \\ Astronomy Program \\ University of Maryland
}

Received SEPTEMRER 22,1972

Running Title: Velocity Gradients and Microturbulence 


\section{ABSTRACT}

Variations of the microturbulent velocity with phase and height in the atmosphere have been reported in classical Cepheids. It is shown that these effects can be understood in terms of variations of the velocity gradient in the atmospheres of these stars. 


\section{INTRODUCTION}

Struve (1932) introduced the concept of microturbulence to explain the anomalously large Doppler broadening velocities he found for supergiants. This was explained by Struve and Elvey (1934) as being due to either a "turbulence of small eddies" or to "several shells which expand with different velocities." It is now known that microturbulence varies with height in the atmospheres of supergiants (Wright 1946, Huang and Struve 1960) and with phase in Cepheids (van Paradijs 1972). Differential motions have been reported in the atmospheres of supergiants of many spectral types (Abt 1957, Ayden 1972, Rosendhal and Wegner 1970) and in particular in Cepheids (van Hoof and Deurinck 1951, Dawe 1969). Van Paradijs (1972) ha's noted that the microturbutence in Cepheids is a maximum near the phase of most rapid contraction. Dawe (1969) and van Hoof and Deurinck (1951) have shown that the velocity gradient is appreciable at this phase. The problem of the effect of a velocity gradient on the curve of growth was investigated by Kubiowski and Ciurla (1965) and Ciurla (1966) for a B2V star. Their work is extended in this paper to a cooler supergiant model. It will be shown that the microturbulence can be explained by a velocity gradient in the atmosphere that is consistent with observed velocities of the spectral lines. 


\section{METHOD}

The method for computing 1 ine profiles in a moving atmosphere follows a suggestion made by Chandrasekhar (1945). The line absorption is described by a Voigt profile, $H(a, u)$, where a is the damping parameter and $u=\left(\lambda-\lambda_{0}\right) / \Delta \lambda_{D}$. When there are velocities in the atmosphere, $\underline{u}$ must be modified to account for the motions of the gas. This can be done by letting $u^{\prime}=u+\mu v \lambda / c \Delta \lambda_{D}$, where $\mu \underline{v}$ is the local velocity of the gas projected onto the line of sight, and using $H\left(a, u^{\prime}\right)$ to compute the line opacity. The specific intensity can be computed from

$$
I_{v}(0, \mu)=\int_{0}^{\infty} B_{v}\left(t_{v}\right) e^{-t_{v} / \mu} d t_{v} / \mu
$$

and then the flux from

$$
F_{\nu}(0)=2 \int_{0}^{1} I_{\nu}(0, \mu) \mu d \mu .
$$

Other methods commonily used, such as the Feautrier method or the quadrature integration of Milne's second equation (Kourganoff 1952), cannot be used since the line opacity is a function of $\mu$. 


\section{RESULTS}

To make the test case as realistic as possible, a model atmosphere from Parsons (1969) and an FeI line were used. The model has $T_{\text {eff }}=6300^{\circ} \mathrm{K}$ and $\log \mathrm{g}=1.8$ while the $4494.57 \AA \mathrm{FeI}$ Jine (excitation potential 2.2 ev) was chosen to give a reasonable variation of the number of absorbers with depth in the atmosphere.

To study a wider range of effects than Ciurla (1966), curves of growth were computed for $\log a=-1,-2,-3$ and microturbulent velocities $\xi=0$ and $5 \mathrm{~km} \mathrm{~s}^{-1}$ by varying the number of absorbers in the line of sight. These curves are given in Figures $1-3$ and table 1. (The curves for $\xi=0$ and $\xi=5 \mathrm{~km} \mathrm{~s}^{-1}$ do not come together at large $n / n_{0}$ because the ordinate is $-\log W / \lambda$ instead of $-\log \left(H / \Delta \lambda_{D^{\circ}}\right)$ Underhill (1947) has shown that a velocity of expansion (or contraction) constant in $\tau$ cannot change the equivalent width, $W$, of a line. Such a velocity field will produce asymmetric line profiles, however, due to the integration over the surface. As a check several profiles were computed with $y(\tau)=20$ and $40 \mathrm{~km} \mathrm{~s}^{-1}$. In no case was the change in $W$ greater than $7 \%$. This change is due to errors in the angle and frequency integration and can be used as a crude estimate of the errors in all these calculations.

Since little is known about the velocity field in the atmosphere of a Cepheid, an arbitrary choice of $\underline{v}(\tau)$ was made, $y(\tau)=-\alpha \log \tau$. This is convenient because it allows a correlation of mean optical 
depth of formation of a line and its observed radial velocity and is nearly linear with geometrical height in the line forming region. An arbitrary constant may be added to $\underline{v}(\tau)$ without changing $W$, but it will change the shape of the profile.

The results for $\xi=0$ and $\alpha=5$ and $10 \mathrm{~km} \mathrm{~s}^{-1}$ are shown in Figures 1-3 and Table 2. With $\alpha=10 \mathrm{~km} \mathrm{~s}^{-1}$ the curve of growth $\left(C_{\alpha 10}\right)$ is nearly identical to the normal curve of growth with $\xi=5 \mathrm{~km} \mathrm{~s}^{-1}$ $\left(c_{\xi 5}\right)$ until the damping portion is reached. In a 17 cases $C_{\alpha 10}$ has a wider plateau than $C_{\xi 5}$. An observer would interpret this as being due to a lower value of the damping parameter $\underline{\text { a. }}$. The decrease in $\underline{a}$ at phases when $\xi$ is large has been observed by Rodgers and Bel1 (1968a, 1968b). This change in a is easily understood. The vertical shift between the damping parts of $C_{\xi 5}$ and $C_{\xi 0}$ is proprotional to the ratio of the Doppler widths. Since $C_{\alpha 10}$ and $C_{\alpha 5}$ have the same Doppler width as $C_{\xi 0}$, in the strong line asymptotic limit the three curves must join. The only way this can happen is for $C_{\alpha 10}$ to be below $C_{\xi 5}$ as the lines get strong.

Ciurla (1966) has done a similar calculation for a B2V model and a line with $\log a \sim-7$. His results agree well with those presented here but, he needed a larger velocity gradient to mimic $\xi=5 \mathrm{~km} \mathrm{~s}^{-1}$. The velocity difference between the strongest and the weakest lines is about $17 \mathrm{~km} \mathrm{~s}^{-1}$ as opposed to the $9 \mathrm{~km} \mathrm{~s}^{-1}$ obtained here. 
Dawe (1969) has plotted observed velocity versus mean optical depth of formation for weak lines in \& Carinae. Reading from Dawe's figure 3 , the weakest 7 ines, formed near $\tau=0.3$, show a velocity of about $18 \mathrm{~km} \mathrm{~s}^{-1}$ while those formed near $\tau=0.1$ show about $22 \mathrm{~km} \mathrm{~s}^{-1}$. \& use $\alpha=15 \mathrm{~km} \mathrm{~s}^{-1}$ to correspond roughty to $\xi=7.5 \mathrm{~km} \mathrm{~s}^{-1}$ observed by Rodgers and Bell (1968a) near this phase. Using $v=-\alpha \log \tau$, $v(0.1)-v(0.3)=7.2 \mathrm{~km} \mathrm{~s}^{-1}$. Correcting for the integration over the surface by the factor $v_{\text {pu }} / s / v_{\text {rad }}=24 / 17$, the predicted velocity difference is $5.0 \mathrm{~km} \mathrm{~s}^{-1}$. The radial velocities "observed" from the minima of the computed profiles are given in Table 3. An exact comparison is not meaningfur since the observed velocities and shapes of the lines are more sensitive to the velocity distribution than is the shape of the curve growth. An attempt to match the observed velocities and profiles more exactly must wait until more is known of the true velocity field. 
IV. CONCLUSIONS

It has been shown that at least part of the microturbulence in Cepheids can be explained by a velocity gradient in the atmosphere that is consistent with the observations. Variations in the velocity gradient with phase and height in the atmosphere can produce the observed variations in microturbulence.

The method can also be applied to normal supergiants to see if their microturbulent velocities can be explained in the same way. Rosendhal (1970) has shown that microturiutence decreases from $A O$ to later spectral types in supergiants and Rosendhal and Wegner (1970) have reported velocities in the atmospheres of $A$ supergiants which they associate with mass ioss. If this interpretation is correct, the oort constant A and the possible expansion term $K$ determined from radial velocities of supergiants will have a systematic error. Due to the expansion of the atmosphere, alt the measured radial velocities will be too small by several $\mathrm{km} \mathrm{s}^{-1}$. Discrepancies between optical and radio determinations of the spiral structure of the galaxy have been discussed and are referenced by Mihalas and Routly (1968). If the variation in microturbulence with spectral type can be interpreted as a variation in the velocity of expansion of the atmosphere, some of the scatter in the determination of the galactic structure constants can be explained. 
I would like to thank Dr. J. P. Harrington and Dr. D. Fischel for their many helpfur comments and Dr. R. A. Bell for bringing several usefur references to my attention. Financial support for this work was provided by NASA Grant NGL-21-002-033. Computer time was included under the budget of the Laboratory for Optical Astronomy, Goddard Space Flight Center.

From a dissertation to be submitted to the Graduate School, University of Maryland, by Alan H. Karp in partial fulfillment of the requirement for the Ph.D. degree in Astronomy. 


\section{9}

\section{REFERENCES}

Abt, H. A. 1957, Ap. J., 126, 138.

Aydin, C. 1972, Astro. \& Ap., 19, 369.

Chandrasekhar, S。 1945, Rev. Mod. Phys., 17, 138.

Ciur1a, T. 1966, Acta Astron., 16, 249.

Dawe, J. A. 1969, M.N.R.A.S., 145, 377.

Hoof, A. van and Deurinck, R. 1951, Ap. J., 115, 116.

Huang, S-S. and Struve, 0. 1960, Stellar Atmospheres, Greenstein, J. L., ed., (Chicago: University of Chicago Press), p. 355.

Kourganoff, V. 1952, Basic Methods in Transfer Problems, (Oxford, England: Clarendon Press), p. 35.

Kubiowski, J。 and Ciurla, T. 1965, Acta Astron., 15, 177.

Mihalas, D. and Routly, P. 1968, Galactic Astronomy, (San Francisco:

H. H. Freeman and Company), p. 147.

Paradijs, J。A. yan 1972, Nature Physical Science, $238,37$.

Parsons, S。 B. 1969, Ap. J. Supp., 18, 127.

Rodgers, A.W. and Be11, R. A. 1968a, M.N.R.A.S., 138, 23

1968 b, M.N.R.A.S., $139,175$.

Rosendha 1, J. D. 1970, Ap. J., 169, 627 .

Rosendhal, J。D. and Wegner, G. 1970, Ap. J., 162, 547.

Underhi17, A. B. 1947, Ap. J., 106, 128.

Wright, Ko O. 1946, J.R.A.S. Canada, 40, 183. 


\section{TABLE 1}

$-\log W / \lambda$ vs $\log$ of Relative Number of Absorbers No Velocity Gradient

\begin{tabular}{lcccccc}
\hline \hline $\left.\log a^{-1}\right)$ & -1 & -1 & -2 & -2 & -3 & -3 \\
$\xi(\mathrm{km} \mathrm{s})$ & 0 & 5 & 0 & 5 & 0 & 5 \\
$\log \mathrm{n} / \mathrm{n}_{0}$ & & & & & & \\
\hline-1.000 & 5.203 & 5.023 & 5.235 & 5.031 & 5.241 & 5.031 \\
-0.699 & 5.060 & 4.795 & 5.110 & 4.811 & 5.117 & 4.812 \\
-0.399 & 4.949 & 4.610 & 5.022 & 4.637 & 5.032 & 4.641 \\
-0.097 & 4.850 & 4.470 & 4.953 & 4.513 & 4.968 & 4.518 \\
0.204 & 4.747 & 4.356 & 4.894 & 4.423 & 4.917 & 4.432 \\
0.505 & 4.634 & 4.257 & 4.833 & 4.354 & 4.874 & 4.368 \\
0.806 & 4.511 & 4.154 & 4.776 & 4.295 & 4.836 & 4.317 \\
1.107 & 4.373 & 4.034 & 4.693 & 4.238 & 4.802 & 4.274 \\
1.408 & 4.234 & 3.922 & 4.616 & 4.179 & 4.768 & 4.237 \\
1.709 & 4.093 & 3.797 & 4.510 & 4.115 & 4.727 & 4.203 \\
2.010 & 3.953 & 3.670 & 4.398 & 4.035 & 4.683 & 4.171 \\
2.311 & - & - & - & - & 4.625 & 4.138 \\
2.612 & - & - & - & - & 4.536 & 4.102 \\
2.913 & - & - & - & - & 4.415 & 3.944 \\
& & & & & & \\
\hline
\end{tabular}


TABLE 2

$-\log W / \lambda$ vs $\log$ of Relative Number of Absorbers $v(\tau)=-\alpha \log \tau$

\begin{tabular}{lcccccc}
\hline \hline $\begin{array}{l}\log a \\
\alpha\left(\mathrm{km} \mathrm{s}^{-1}\right)\end{array}$ & -1 & -1 & -2 & -2 & -3 & -3 \\
$\log n / n_{0}$ & & 10 & 5 & 10 & 5 & 10 \\
\hline-1.000 & 5.138 & 4.994 & 5.099 & 5.039 & 5.091 & 5.040 \\
0.204 & 4.528 & 4.392 & 4.608 & 4.429 & 4.621 & 4.432 \\
1.408 & 4.174 & 4.083 & 4.417 & 4.222 & 4.454 & 4.240 \\
2.612 & 3.666 & 3.635 & 4.119 & 4.027 & 4.334 & 4.747 \\
\hline
\end{tabular}

\section{TABLE 3}

Radial Velocity in $\mathrm{km} \mathrm{s}^{-1}$ Observed from Computed Profiles for Cases in Table 2

\begin{tabular}{lrrrrrr}
\hline \hline $\log a$ & -1 & -1 & -2 & -2 & -3 & -3 \\
$\alpha\left(\mathrm{km} \mathrm{s}^{-1}\right)$ & 5 & 10 & 5 & 10 & 5 & 10 \\
$\log \eta / n_{0}$ & & & & & & \\
\hline-1.000 & 2.9 & 5.4 & 3.3 & 5.4 & 2.7 & 5.4 \\
0.204 & 5.4 & 6.6 & 5.4 & 9.3 & 4.2 & 9.3 \\
1.408 & 6.7 & 13.3 & 6.6 & 14.7 & 6.6 & 14.7 \\
2.612 & 10.8 & 14.7 & 9.3 & 14.7 & 6.6 & 14.7 \\
\hline
\end{tabular}


CAPTIONS TO FIGURES

Fig. 1. Curves of growth for $\log a=-1 . \quad-, \quad \xi=0\left(C_{\xi 0}\right)$;

$\longrightarrow, \xi=5 \mathrm{~km} \mathrm{~s}^{-1}\left(C_{\xi 5}\right) ; 0 \longrightarrow 0, \alpha=5 \mathrm{~km} \mathrm{~s}^{-1}$

$\left(C_{\alpha 5}\right) ; \bullet, \alpha=10 \mathrm{~km} \mathrm{~s}^{-1}\left(C_{\alpha 10}\right) . \xi$ is the micro-

turbulent velocity and $\alpha$ is the velocity gradient parameter.

Fig. 2. Curves of growth for $\log a=-2$. Notation is the same as in Figure 1.

Fig. 3. Curves of growth for $\log a=-3$. Notation is the same as in Figure 1. 
13

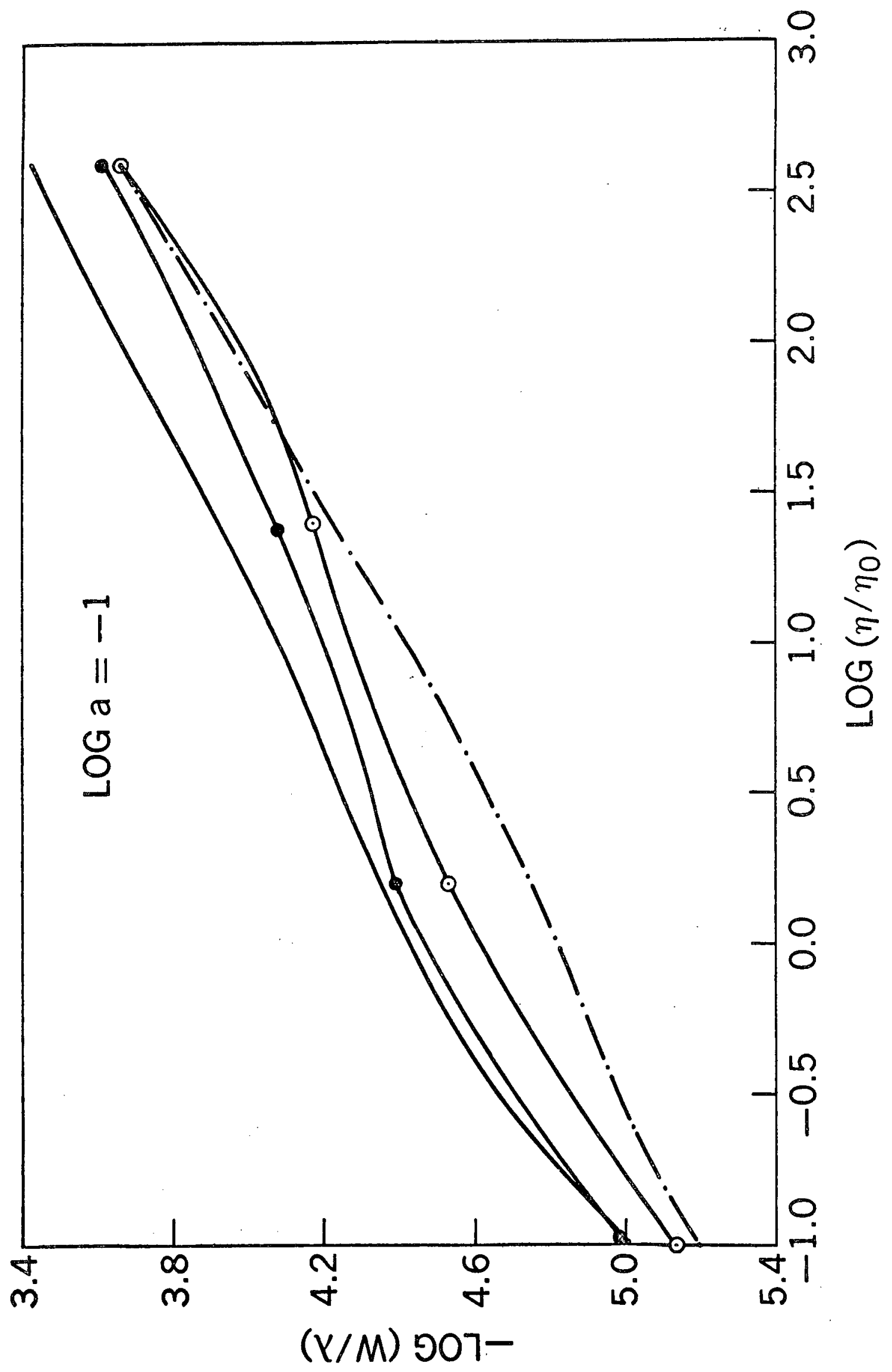




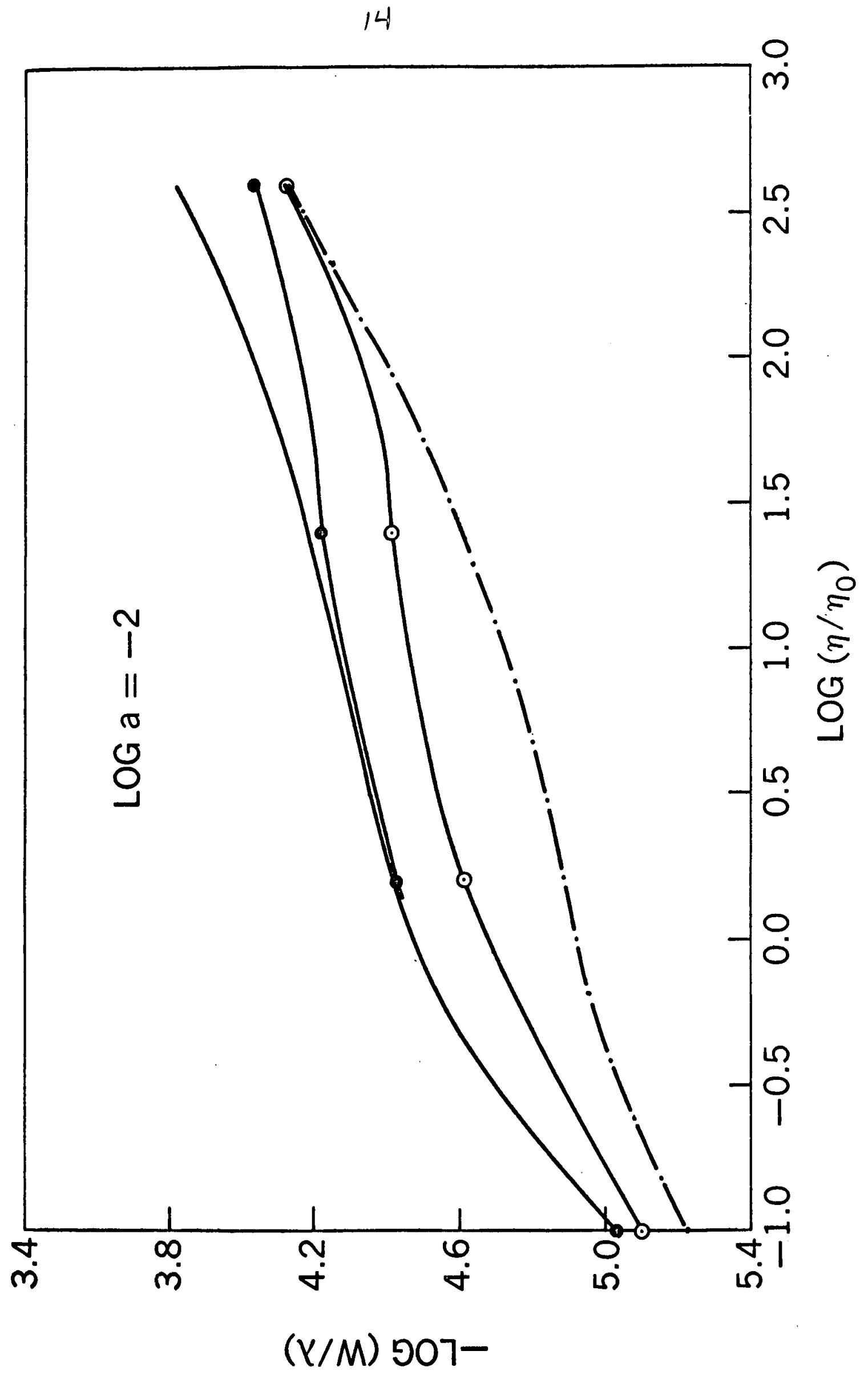




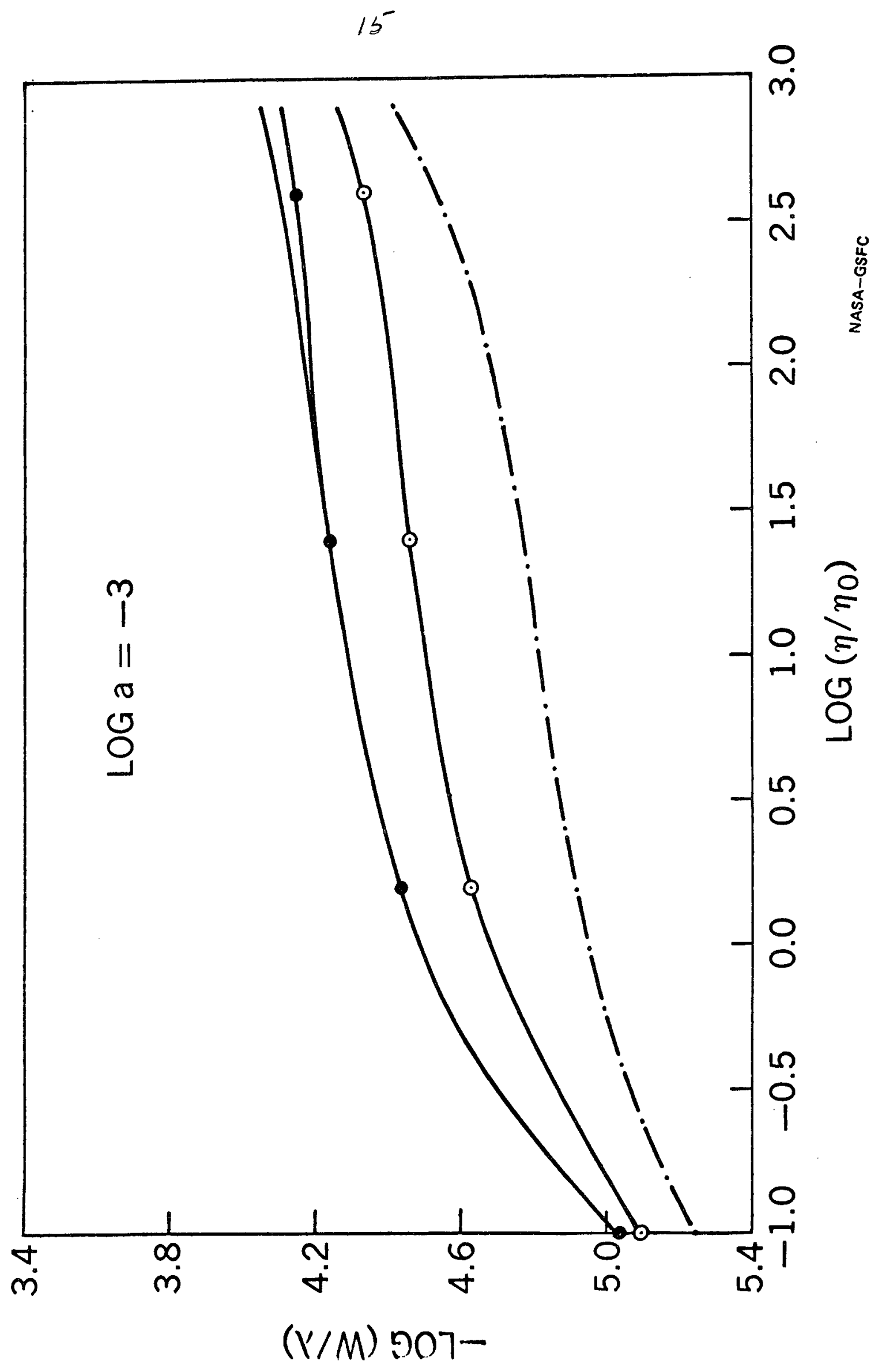

